\title{
La fin du Pléistocène moyen et le début du Pléistocène supérieur dans la moyenne vallée du Rhône
}

\section{Marie-Hélène Moncel}

\section{(2) OpenEdition \\ Journals}

Édition électronique

URL : http://journals.openedition.org/adlfi/1860

ISSN : 2114-0502

Éditeur

Ministère de la culture

Référence électronique

Marie-Hélène Moncel, « La fin du Pléistocène moyen et le début du Pléistocène supérieur dans la moyenne vallée du Rhône ", ADLFI. Archéologie de la France - Informations [En ligne], Rhône-Alpes, mis en ligne le 01 mars 2008, consulté le 26 avril 2019. URL : http://journals.openedition.org/adlfi/1860

Ce document a été généré automatiquement le 26 avril 2019

(c) Ministère de la Culture et de la Communication, CNRS 


\title{
La fin du Pléistocène moyen et le début du Pléistocène supérieur dans la moyenne vallée du Rhône
}

\author{
Marie-Hélène Moncel
}

Date de l'opération : 2007 - 2008 (PT)

1 La campagne 2007 a poursuivi le programme tri-annuel débuté en 2005 sur les remplissages attribués à la fin du Pléistocène moyen et au début du Pléistocène supérieur dans la moyenne vallée du Rhône, afin de préciser le cadre chronologique et biostratigraphique des occupations du Paléolithique moyen, cadre encore souvent mal connu. Deux sites ont été l'objet de cette campagne : la grotte du Figuier à Saint-Martin d'Ardèche et le secteur de Chazalis à Beaulieu.

\section{Grotte du Figuier à Saint-Martin-d'Ardèche}

2 La grotte s'ouvre actuellement à $39 \mathrm{~m}$ au dessus de l'Ardèche, sur sa rive gauche, au pied d'une falaise calcaire du Crétacé inférieur. Les premières fouilles menées par L. Chiron et P. Raymond ont porté sur le fond et le centre de la cavité. Mais c'est à P. et A. Huchard, A. Obenich, M. Veyrier, R. Ribeyre, R. Gilles et J. Combier que reviennent les travaux sur le site à partir des années 1950 .

3 Une séquence attribuée de la fin du Würm II à la fin du Würm IV a été décrite, en particulier par J. Combier, mais aucune donnée radiométrique ne le confirme actuellement. La seule coupe disponible est une synthèse relevée par $\mathrm{M}$. Veyrier, à la jonction de la salle 1 et de l'entrée du couloir ouest menant à la salle 2. Deux niveaux moustériens, en particulier, ont été observés à la base, dont un niveau de type Quina avec des faunes froides, reposant sur un sable micacé stérile (Combier, 1967 ; Moncel, 2001). Un niveau stérile sépare de nouveau ces deux couches du reste de la séquence qui regroupe des niveaux du Paléolithique supérieur, de l'Aurignacien (Woerz, 2006) au Magdalénien. 
4 Alors que la salle 1 se présente comme une large cavité de $150 \mathrm{~m}^{2}$ environ et de $10 \mathrm{~m}$ de haut, ouvert par un porche de $15 \mathrm{~m}$ de large, le couloir qui démarre à l'ouest s'étend sur environ $25 \mathrm{~m}$. Très bas de plafond $(50 \mathrm{~cm}$ à $1 \mathrm{~m})$, il est barré par des planchers stalagmitiques en encorbellement, dégagés par l'érosion. Une diaclase, dans la salle 3, permet de communiquer avec l'extérieur et il est probable qu'une autre entrée devait exister pour accéder à cette salle qui est séparée de la salle 2 par une étroite chatière.

Alors que la séquence de la salle 1 paraît avoir été bien reconnue (plusieurs niveaux de sables et de cailloutis), celles du couloir ouest menant à la salle 2, puis à la salle 3 , sont encore mal connues. Le couloir a été désobstrué par les frères Huchard et leur équipe en 1947 et 1948 (inscriptions sur le plafond de la salle 2). La couche aurignacienne serait bien distincte des niveaux moustériens dans la salle 2 et dans le couloir, moins nettement dans la salle 3. La brèche qui bouchait l'entrée du couloir englobait à la fois des niveaux du Solutréen et du Magdalénien selon J. Combier. C'est aussi dans cette zone qu'une sépulture d'enfant a été dégagée au milieu d'un chaos de blocs. Son âge (Paléolithique supérieur ?) fait encore l'objet de discussions (Veyrier et al., 1953 ; Billy, 1979). Une autre calotte crânienne aurait été découverte dans la salle 3.

La salle 1 ayant été vidée par les fouilles anciennes, les travaux ont porté principalement sur les salles 2 et 3 dans l'espoir de retrouver des lambeaux de stratigraphie. Le couloir de jonction entre les salles 1 et 2 a fait l'objet d'un diagnostic et est apparu très perturbé en surface (test de $30 \mathrm{~cm}$ de profondeur de déblaiement pour sortir les sédiments de la fouille).

7 Outre les sondages effectués dans les salles 2 et 3 , un travail de déblais dans la salle 1 , à l'aplomb de la paroi ouest sous le plancher stalagmitique, a permis de retrouver en place les sables micacés et de dégager des brèches collées contre la paroi. De même, un sondage dans les déblais de la terrasse a permis de dégager une brèche stérile se raccordant à celle visible sur les parois extérieures. Le tamisage a permis de retrouver des artéfacts. Le sondage de la salle 2 offre une séquence composée de quatre ensembles lithostratigraphiques :

$91 /$ couche remaniée avec fragments de plancher stalagmitique (anciennes fouilles) de 50 à $60 \mathrm{~cm}$;

102 / ensemble d'argile brun caillouteux de $40 \mathrm{~cm}$ d'épaisseur ;

113 / couche sableuse et argileuse rouge peu caillouteuse de 15-20 cm d'épaisseur ;

124 / sable de base avec croûtes ferrugineuses (plus de 1,20 m sans atteindre le substrat).

13 Le sondage de la salle 3 est plus complexe et paraitt montrer que les fouilles anciennes ont certainement perturbé très profondément le remplissage :

$141 /$ couche remaniée de $80 \mathrm{~cm}$ au moins (reste d'une hache rouillée à $80 \mathrm{~cm}$ de pronfondeur);

2 / argile brune sableuse avec rares cailloutis de $20 \mathrm{~cm}$ d'épaisseur ;

3 / sable de base fortement micacé (plus de 1,20 $\mathrm{m}$ sans atteindre le substrat).

L'identification de niveaux archéologiques est particulièrement difficile en l'état. Du matériel est présent sur toute la séquence, tant lithique qu'osseux. En effet, outre les niveaux supérieurs remaniés, et les niveaux argilo-sableux de base, il n'est pas certain que la couche intermédiaire à cailloutis abondants dans la salle 2 soit totalement en place. Le matériel est réparti sur toute la couche. 
18 L'abondance des restes de carnivores et d'ursidés pose la question de l'utilisation du réseau intérieur karstique comme tanière lors ou après des occupations humaines. Des prélèvements sédimentologiques et micromorphologiques permettront de discuter sur la mise en place de la base des deux séquences. L'étude du matériel archéologique, qui est en cours, apportera aussi sans doute quelques réponses, bien qu'un premier tri laisse penser à un mélange d'artefacts (production d'éclats, production laminaire). Les couches archéologiques identifiées par les fouilles anciennes (Combier, 1967) n'ont pas été reconnues dans les sondages.

\section{Secteur de Chazalis à Beaulieu}

19 Sur la rive droite de la moyenne vallée du Rhône, les témoignages relevant de l'Acheuléen sont rares et le site d'Orgnac 3 est le seul gisement présentant une séquence datée des stades isotopiques 9 et début 8 . A Chazalis, un biface a été découvert en surface il y a plus de 20 ans. Le contexte géologique est une ancienne terrasse du Chassezac, situé actuellement au nord de ce secteur. Un sondage dans la terrasse a permis de reconnaître la séquence sur une épaisseur de 2,40 m. Celle-ci est malheureusement stérile, composée d'un sol humifère, puis d'un niveau sableux de plus de $1 \mathrm{~m}$ d'épaisseur, puis de sables et de cailloutis abondant et enfin d'un substrat calcaire très concassé.

\section{Année 2008}

Cette année 2008 est une année complémentaire d'un programme tri-annuel qui a débuté en 2005. Les objectifs étaient de mener à bien des prospections dans la région et préciser les données sur plusieurs séquences de référence du Paléolithique moyen.

21 Les prospections se sont poursuivies, en particulier dans les secteurs de la vallée du Rhône, des gorges de l'Ardèche et sur le plateau de Cruas. Elles ont permis de compléter les échantillonnages en matières premières et de repérer d'anciens lieux de découvertes.

D’autres travaux se sont également déroulés sur les sites du Figuier et du Ranc Pointu n ${ }^{\circ}$ 2.

23 La grotte du Figuier avait déjà fait l'objet d'une première campagne en 2007, en particulier dans les salles profondes 3 et 2, permettant d'identifier le remplissage et de préciser les types d'occupations humaines et animales du karst profond. A cette occasion, des dépôts indurés encore en place avaient été identifiés dans la première salle, en bordure de la paroi, à proximité d'un petit diverticule comblé et au contact des sables stériles. Ces dépôts ont fait l'objet d'un ravivage lors de la campagne 2008, ce qui a permis d'identifier des niveaux archéologiques meubles encore en place et de les fouiller sur 30 $\mathrm{cm}$ de profondeur et $60 \mathrm{~cm}$ de hauteur. A la base, du matériel lithique, pouvant être rapporté au Paléolithique moyen, a été dégagé. Au sommet, ce sont quelques grandes et fines lames brutes qui ont été découvertes, permettant de supposer que nous avons atteint un niveau du Paléolithique supérieur (?). L'observation des sédiments conservés en bordure de la paroi indique que seule une partie de la séquence archéologique a été conservée. D'après les données issues des travaux antérieurs, il pourrait s'agir pour les niveaux du Paléolithique supérieur de niveaux magdaléniens. Le matériel osseux est abondant, bien que très fragmentés, composés de restes de rennes et chevaux. Une dent de cheval, bien conservée et issue des niveaux de base, est datable. Si cette datation 
s'avère possible, elle permettra de donner pour la première fois un âge aux premiers niveaux d'occupation de la salle 1, en l'occurrence un Moustérien de type Quina.

Situé sur la même vire que Le Figuier, la grotte du Ranc Pointu n 2 a livré dans le passé une petite séquence avec au moins un niveau d'occupation humaine. Une étude stratigraphique et sédimentologique y avait été effectuée par E. Debard. La coupe, bien conservée, a été reculée sur une petite superficie. Les grandes étapes du remplissage ont été de nouveau identifiées et le lit de galets stérile de la base a été atteint. Le niveau archéologique a été localisé. Des échantillonnages micromorphologiques et palynologiques ont été prélevés dans les deux grottes.

25 A l'issue de ce programme, il est maintenant possible de proposer des données nouvelles sur le cadre bio et chronostratigraphique des sites étudiés (abri du Maras, baume Flandin, abri des Pêcheurs, Le Figuier et Ranc Pointu n ${ }^{\circ} 2$ ) et de les replacer les uns par rapport aux autres entre les stades isotopiques 5 et 4 . Par ailleurs, l'étude a été couplée avec une analyse des comportements humains, tant au niveau technique que de subsistance, permettant de discuter sur la variabilité des stratégies humaines dans le temps et selon les conditions climatiques.

Les prospections ont permis d'alimenter la base de données sur les gîtes en silex qui couvre maintenant toute les marges sud-est du Massif central, en collaboration avec Paul Fernandes et Jean-Paul Raynal. De nombreuses localités de plein air ont été de nouveau localisées, quelques nouvelles ont été découvertes, enrichissant le maillage des sites du Paléolithique moyen dans la région.

\section{INDEX}

Index chronologique : Paléolithique moyen, Pléistocène

Index géographique : Rhône-Alpes, Ardèche (07)

operation Prospection thématique (PRT)

\section{AUTEUR}

\section{MARIE-HÉLÈNE MONCEL}

Museum national d'histoire naturelle 